\title{
Solving Fredholm integral equations of the first kind by using wavelet bases
}

\author{
Yaser Rostami ${ }^{1}$ (D), Khosrow Maleknejad ${ }^{* 2}$ (D) \\ ${ }^{1}$ Department of Engineering, Malard Branch, Islamic Azad University, Tehran, Iran \\ ${ }^{2}$ School of Mathematics, Iran University of Science and Technology, Narmak, Tehran, 16846, Iran
}

\begin{abstract}
In this paper, we used a project technique for solving integral equation of the first kind by wavelet families via regularization approach and we proved the convergence for the numerical method and error consideration. Semi-orthogonal B-spline scaling functions and wavelets of degree 4 and their dual functions are presented to approximate the solutions to integral equations. Sparse matrix will product of semi-orthoganality and vanishing moment properties of B-spline wavelets.
\end{abstract}

Mathematics Subject Classification (2010). 45B05, 65R20

Keywords. Fredholm integral equations, projection method, regularization, quartic B-spline wavele

\section{Introduction}

One of the inverse problems is the Fredholm integral equations of the first kind (FK1) that arise in many areas of science and engineering fields such as image processing and electromagnetic. The below equation that can be written in the generic form is the FK1 with a square integrable kernel:

$$
(K \phi)(x)=\int_{a}^{b} k(x, s) \phi(s) d s=f(x), a \leq x \leq b .
$$

To classify integral equations, we denote the unknown function by $\phi(x)$, the kernel of the equation by $k(x, s)$, and the free term, which is assumed known, by $f(x)$. We introduce the integral operator $K$ defined by $K \phi=f$.

A FK1 is of the form (1.1) where the functions $\phi(x)$ and $f(x)$ are assumed to belong to the class $L_{2}[a, b]$, since the integral operator $K$ with a non-degenerate and continuous kernel $k(x, s)$ is a compact operator with the non-closed range in $L_{2}[a, b]$ and hence it is not continuously invertible [2].

The wavelets technique allows the creation of very fast algorithms when compared with known algorithms $[7,8]$. Various wavelet bases are applied in order to solve (1.1) Sinc collocation [14], Legendre wavelet [9], Chebyshev wavelet [1], wavelets-Galerkin method [6], multiwavelet [12], B-spline wavelet [11].

\footnotetext{
*Corresponding Author.

Email addresses: rostamiysr@iaumalard.ac.ir (Y. Rostami), maleknejad@iust.ac.ir (K. Maleknejad)

Received: 03.02.2017; Accepted: 27.06.2018
} 
The layout of the paper is as follows: Section 2 is devoted to the quartic (five order) Semi-Orthogonal (SO) B-spline Wavelets Collocation (BSWC). In Section 3, the method of regularization is used to approximate the solution of (1.1). As a result, a set of algebraic equations is achieved and the solution of the considered problem is introduced. In Section 4 , error and convergence consideration of regularization method and theorems are proven and is shown that the procedure converges to the solution. In the last section, illustrative examples are given to show accuracy, validity and applicability of the numerical technique.

\section{B-spline scaling functions and wavelets on $[0,1]$}

We can generate a doubly-indexed family of wavelets form $\psi$ by dilating and translating [4]:

$$
\psi_{a, b}(x)=|a|^{-\frac{1}{2}} \psi\left(\frac{x-b}{a}\right), a \neq 0, a, b \in \mathbb{R} .
$$

The wavelets have been grouped in different families. Generally, we can say that jth generation of daughters will have $2^{j}$ wavelets defined by:

$$
\psi_{j, k}(x)=\psi\left(2^{j} x-k\right) ; 0 \leq k \leq 2^{j}-1 ; j, k \in \mathbb{Z} .
$$

The members of this generation will be constant on intervals of length $2^{-(j+1)}$. The first idea in studying of wavelets was this matter that we can write functions as linear combinations of the Father and Mother wavelets and first generation of daughters. This basis denoted by $B_{j}$. There is another basis of wavelets that is called sons wavelet. Here, we can define generations of sons wavelet by the following relation :

$$
\varphi_{j, k}(x)=\psi\left(2^{j} x-k\right) ; 0 \leq k \leq 2^{j}-1 ; j, k \in \mathbb{Z},
$$

which assume $S_{j}$ denote the set of $2^{j}$ functions $\left\{\varphi_{j, k}(x)\right\}_{k=0}^{2^{j}-1}$.

Therefore, $S_{j}$ will account as a basis for the inner product space $V_{j}$. Vector space $V_{j}$ with the basis $S_{j}$ forms a nested sequence of subspaces $V_{0} \subseteq V_{1} \subseteq V_{2} \subseteq \ldots$ and uses the basis $B_{j}$ for $V_{j}$ and orthogonal decomposition theorem. Therefore we will have:

$$
V_{j}=V_{j-1} \oplus V_{j-1}^{\perp}=\left(V_{j-2} \oplus V_{j-2}^{\perp}\right) \oplus V_{j-1}^{\perp}=\ldots=V_{0} \oplus V_{0}^{\perp} \oplus V_{1}^{\perp} \oplus \ldots V_{j-1}^{\perp} .
$$

The wavelets have especial particularities that all of them are gathered in a collection of Multi-Resolution Analysis (MRA) on $L^{2}(\mathbb{R})$ which is defined as a sequence of closed subspaces $V_{j}$ with the following properties ([3],page 180):

(1) $V_{j} \subset V_{j+1}$.

(2) $f(x) \in V_{j} \Longleftrightarrow f(2 x) \in V_{j+1}$.

(3) $f(x) \in V_{0} \Longleftrightarrow f(x+k) \in V_{0}$.

(4) $\bigcup V_{j}$ is dense in $L^{2}(\mathbb{R})$ and $\bigcap V_{j}=0$.

(5) A scaling function $\varphi \in V_{0}$, exists such that the collection $\varphi$ is Riesz basis of $V_{0}$.

Some of the important properties relevant to the present work are given below:

1. vanishing moments: A wavelet is said to be have a vanishing moment of order $m$ if

$$
\int_{-\infty}^{+\infty} x^{p} \psi(x) d x=0 ; \quad p=0, \ldots, m-1
$$

all wavelets must satisfy the above condition for $p=0$.

2. semi-orthogonality: The wavelets $\psi_{j, k}$ form a semi-orthogonal basis if

$$
\left\langle\psi_{j, k}, \psi_{i, s}\right\rangle=0 ; \quad i \neq j ; \quad \forall i, j, k, s \in \mathbb{Z}
$$

the generalization to biorthogonal wavelets has been considered to gain more flexibility. Here, a dual scaling function $\tilde{\varphi}$ and a dual wavelet $\tilde{\psi}$ exist that generate a Dual MultiResolution Analysis (DMRA) with subspaces $\widetilde{V}_{j}$ and $\widetilde{V_{j}^{\perp}}$, such that:

$$
\widetilde{V}_{j} \perp V_{j}^{\perp} \text { and } V_{j} \perp \widetilde{V_{j}^{\perp}} \text {, }
$$


and consequently

$$
\widetilde{V_{j}^{\perp}} \perp V_{j^{\prime}}^{\perp} \text { for } j \neq j^{\prime} .
$$

When semi-orthogonal wavelets are constructed from B-spline of order $m$, the lowest octave level $j=j_{0}$ is determined in [3] by

$$
2^{j_{o}} \geq 2 m-1,
$$

so as to give a minimum of one complete wavelet on the interval $[0,1]$. In this paper we used a wavelet generated by a quartic $(m=5)$ cardinal B-spline function.

From (2.1), the five order B-spline lowest level, which must be an integer, is determined to $j_{0}=4$. For each level $j \geq j_{0}$ this constrains all octave levels to $j \geq 4$.

Definition 2.1. Let $m$ and $n$ be two positive integers and ([5, page 236])

$$
a=x_{-m+1}=\ldots=x_{0}<x_{1}<\ldots<x_{n}=x_{n+1}=\ldots=x_{n+m-1}=b,
$$

be an equally spaced knots sequence. The functions

$$
B_{m, j, X}(x)=\frac{x-x_{j}}{x_{j+m-1}-x_{j}} B_{m-1, j, X}(x)+\frac{x_{j+m}-x}{x_{j+m}-x_{j+1}} B_{m-1, j+1, X}(x),
$$

and

$$
B_{1, j, X}(x)=\left\{\begin{array}{cc}
1 & x \in\left[x_{j}, x_{j+1}\right), \\
0 & \text { else, }
\end{array}\right.
$$

are called cardinal B-spline functions of order $m \geq 2$ for the knot sequence $X=$ $\left\{x_{k}^{j}\right\}_{k=1-m}^{2^{j}+m-1}$ and $\operatorname{Supp}\left[B_{m, j, X}(x)\right]=\left[x_{j}, x_{j+m}\right] \cap[a, b]$.

For the sake of simplicity, suppose $[a, b]=[0, n]$ and $x_{k}=k, k=0, \ldots n$. The $B_{m, j, X}(x)=B_{m}(x-j), j=0, \ldots, n-m$ are interior B-spline functions, while the remaining $j=-m+1, \ldots,-1$ and $j=n-m+1, \ldots, n-1$ are boundary B-spline functions. Since the boundary B-spline functions at 0 are symmetric reflections of those at $n$, it is sufficient to construct only the first half functions by simply replacing $x$ with $n-x$.

By considering the interval $[a, b]=[0,1]$, at any level $j \in \mathbb{Z}^{+}$, the discrete step is $2^{-j}$, and this generates $n=2^{j}$ number of segments in $[0,1]$ with knot sequence

$$
X^{j}=\left\{\begin{array}{c}
x_{1-m}^{j}=x_{2-m}^{j}=\ldots=x_{0}^{j}=0, \\
x_{k}^{j}=k 2^{-j} \\
x_{2^{j}}^{j}=x_{2^{j}+1}^{j}=\ldots=x_{2^{j}+m-1}^{j}=0,
\end{array} \quad k=1, \ldots, 2^{j}-1,\right.
$$

For each level $j \geq j_{0}$ the scaling function of order $m$ can be defined as allows:

$$
\varphi_{m, j, k}(x)=\left\{\begin{array}{cc}
B_{m, j_{0}, k}\left(2^{j-j_{o}} x\right) & k=1-m, \ldots,-1, \\
B_{m, j_{0}, 2^{j}-m-k}\left(1-2^{j-j_{o}} x\right) & k=2^{j}-m+1, \ldots, 2^{j}-1, \\
B_{m, j_{0}, 0}\left(2^{j-j_{o}} x-2^{-j_{o}} k\right) & k=0, \ldots, 2^{j}-m
\end{array}\right.
$$

and the two-scale relation for the m-order semi-orthogonal compactly supported B-spline Wavelet (BSW) are defined as follows:

$$
\begin{gathered}
\psi_{m, j, k-m}(x)=\sum_{j=k}^{2 k+2 m-2} q_{k, j} B_{m, j, k-m}(x), k=1, \ldots, m-1, \\
\psi_{m, j, k-m}(x)=\sum_{j=2 k-m}^{2 k+2 m-2} q_{k, j} B_{m, j, k-m}(x), k=m, \ldots, n-m+1, \\
\psi_{m, j, k-m}(x)=\sum_{j=2 k-m}^{n+k+m-1} q_{k, j} B_{m, j, k-m}(x), k=n-m+2, \ldots, n,
\end{gathered}
$$

where $q_{k, j}=q_{j-2 k}$. 
Hence, there are $2(m-1)$ boundary wavelets and $(n-2 m+2)$ inner wavelet in interval $[a, b]$. Finally by considering the level $j$ with $j \geq j_{0}$, the B-spline wavelet in $[0,1]$ can be expressed as follows:

$$
\psi_{m, j, k}(x)=\left\{\begin{array}{cc}
\psi_{m, j_{0}, k}\left(2^{j-j_{o}} x\right) & k=-m+1, \ldots,-1, \\
\psi_{m, j_{0}, 2^{j}-2 m+1-k}\left(1-2^{j-j_{o}} x\right) & k=2^{j}-2 m+2, \ldots, 2^{j}-m, \\
\psi_{m, j_{0}, 0}\left(2^{j-j_{o}} x-2^{-j_{o}} k\right) & k=0, \ldots, 2^{j}-2 m+1,
\end{array}\right.
$$

The scaling functions $\varphi_{m, j, k}(x)$,occupy $m$ segments and the wavelet functions $\psi_{m, j, k}(x)$ occupy $2 m-1$ segments.

\subsection{General order B-spline wavelets}

The B-spline wavelet can be defined recursively by the convolution ([5, page 235]):

$$
\varphi_{m}(x)=\int_{-\infty}^{+\infty} \varphi_{m-1}(x-t) \varphi_{1}(t) d t=\int_{0}^{1} \varphi_{m-1}(x-t) d t
$$

where

$$
\varphi_{1}(x)=\left\{\begin{array}{cc}
1 & 0 \leq x<1 \\
0 & \text { else }
\end{array}\right.
$$

The construction of the scaling function of m-order B-spline is based on the two scale relation:

$$
\varphi_{m}(x)=\sum_{k=0}^{m} p_{k} \varphi_{m}(2 x-k),
$$

where $p_{k}$ is the two scale sequence and can be expressed as a combination:

$$
p_{k}=2^{1-m}\left(\begin{array}{c}
m \\
k
\end{array}\right), \quad 0 \leq k<m .
$$

The two-scale relation for m-order BSW is given by:

$$
\begin{gathered}
\psi_{m}(x)=\sum_{k=0}^{3 m-2} q_{k} \varphi_{m}(2 x-k), \\
q_{k}=(-1)^{k} 2^{1-m} \sum_{l=0}^{m}\left(\begin{array}{c}
m \\
l
\end{array}\right) \varphi_{2 m}(k-l+1), \quad 0 \leq k<3 m-2 .
\end{gathered}
$$

The decomposition relation for m-order BSW is:

$$
\varphi_{m}(2 x-l)=\sum_{k \in \mathbb{Z}}\left(a_{l-2 k} \varphi(x-k)+b_{l-2 k} \psi(x-k)\right), \quad l \in \mathbb{Z},
$$

where decomposition coefficients sequences $\left\{a_{k}\right\}$ and $\left\{b_{k}\right\}$ are as follows:

$$
\begin{aligned}
& a_{k}=\frac{(-1)^{k+1}}{2} \sum_{l} q_{-k+2 m-2 l-1} c_{l, 2 m}, \\
& b_{k}=-\frac{(-1)^{k+1}}{2} \sum_{l} p_{-k+2 m-2 l-1} c_{l, 2 m} .
\end{aligned}
$$

In (2.4) and (2.5) the coefficients sequence $\left\{c_{k, m}\right\}$ is presented by m-th order fundamental cardinal spline functions:

$$
T_{m}(x)=\sum_{k \in \mathbb{Z}} c_{k, m} \varphi_{m}\left(x+\frac{m}{2}-k\right) .
$$




\subsection{Quartic B-spline wavelet $(m=5)$}

Quartic B-spline scaling function $\varphi_{5}(x)$ is given by the next recursive relation:

$$
\varphi_{5}(x)=\left\{\begin{array}{cc}
\frac{x^{4}}{24} & 0 \leq x<1, \\
-\frac{x^{4}}{6}+\frac{5 x^{3}}{6}-\frac{5 x^{2}}{4}+\frac{5 x}{6}-\frac{5}{24} & 1 \leq x<2, \\
\frac{x^{4}}{4}-\frac{5 x^{3}}{2}+\frac{35 x^{2}}{4}-\frac{25 x}{2}+\frac{155}{24} & 2 \leq x<3, \\
-\frac{x^{4}}{6}+\frac{5 x^{3}}{2}-\frac{55 x^{2}}{4}+\frac{65 x}{2}-\frac{655}{24} & 3 \leq x<4, \\
\frac{x^{4}}{24}-\frac{5 x^{3}}{6}+\frac{25 x^{2}}{4}-\frac{125 x}{6}+\frac{625}{24} & 4 \leq x<5, \\
0 & \text { else, }
\end{array}\right.
$$

where the compact support in the range $[0, m]$ referring to the property B-spline scaling functions. Two scale sequences $\left\{p_{k}\right\}_{k=0}^{5}$ and $\left\{q_{k}\right\}_{k=0}^{13}$ are as follow. Based on them, two scale relations for $\varphi_{5}(x)$ and $\psi_{5}(x)$ can be constructed using (2.2) and (2.3) respectively:

$$
\begin{gathered}
\left\{p_{k}\right\}_{k=0}^{5}=\left\{\frac{1}{16}, \frac{5}{16}, \frac{5}{8}, \frac{5}{8}, \frac{5}{16}, \frac{1}{16}\right\}, \\
\left\{q_{k}\right\}_{k=0}^{13}=\left\{\frac{1}{5806080},-\frac{169}{1935360}, \frac{2141}{725760},-\frac{5197}{181440}, \frac{149693}{1161216},-\frac{54289}{165888},\right. \\
\left.\frac{74339}{145152},-\frac{74339}{145152}, \frac{54289}{165888},-\frac{149693}{1161216}, \frac{5197}{181440},-\frac{2141}{725760}, \frac{169}{1935360}, \frac{1}{5806080}\right\} .
\end{gathered}
$$

So the corresponding scaling function is:

$$
\varphi_{j, k}(x)=\left\{\begin{array}{cc}
\frac{\left(x_{j}-k\right)^{4}}{24} & k \leq x_{j}<k+1, \\
-\frac{\left(x_{j}-k\right)^{4}}{6}+\frac{5\left(x_{j}-k\right)^{3}}{6}-\frac{5\left(x_{j}-k\right)^{2}}{4}+\frac{5\left(x_{j}-k\right)}{6}-\frac{5}{24} & k+1 \leq x_{j}<k+2, \\
\frac{\left(x_{j}-k\right)^{4}}{4}-\frac{5\left(x_{j}-k\right)^{3}}{2}+\frac{35\left(x_{j}-k\right)^{2}}{4}-\frac{25\left(x_{j}-k\right)}{2}+\frac{155}{24} & k+2 \leq x_{j}<k+3, \\
-\frac{\left(x_{j}-k\right)^{4}}{6}+\frac{5\left(x_{j}-k\right)^{3}}{2}-\frac{55\left(x_{j}-k\right)^{2}}{4}+\frac{65\left(x_{j}-k\right)}{2}-\frac{655}{24} & k+3 \leq x_{j}<k+4, \\
\frac{\left(x_{j}-k\right)^{4}}{24}-\frac{5\left(x_{j}-k\right)^{3}}{6}+\frac{25\left(x_{j}-k\right)^{2}}{4}-\frac{125\left(x_{j}-k\right)}{6}+\frac{625}{24} & k+4 \leq x_{j}<k+5, \\
0 & \text { else, }
\end{array}\right.
$$

with the respective left and right hand side boundary scaling function, the actual coordinate position $x$ is related to $x_{j}$ according to $x_{j}=2^{j} x$, then we have:

$$
\begin{aligned}
& \varphi_{5}(2 x-k)=\left\{\begin{array}{cc}
\frac{(2 x-k)^{4}}{24} & k / 2 \leq x<k / 2+1 / 2, \\
-\frac{(2 x-k)^{4}}{6}+\frac{5(2 x-k)^{3}}{6}-\frac{5(2 x-k)^{2}}{4}+ & k / 2+1 / 2 \leq x<k / 2+1, \\
\frac{5(2 x-k)}{6}-\frac{5}{24} & k / 2+1 \leq x<k / 2+3 / 2, \\
\frac{(2 x-k)^{4}}{4}-\frac{5(2 x-k)^{3}}{2}+\frac{35(2 x-k)^{2}}{4}- & \\
\frac{25(2 x-k)}{2}+\frac{155}{24} & k / 2+3 / 2 \leq x<k / 2+2, \\
-\frac{(2 x-k)^{4}}{6}+\frac{5(2 x-k)^{3}}{2}-\frac{5(2 x-k)^{2}}{4}+ & \\
\frac{65(2 x-k)}{2}-\frac{655}{24} & k / 2+2 \leq x<k / 2+5 / 2, \\
\frac{(2 x-k)^{4}}{24}-\frac{5(2 x-k)^{3}}{6}+\frac{25(2 x-k)^{2}}{4}- & \text { else, }
\end{array}\right. \\
& \varphi_{5}(x)=\frac{1}{16} \varphi(2 x)+\frac{5}{16} \varphi(2 x-1)+\frac{5}{8} \varphi(2 x-2)+ \\
& \frac{5}{8} \varphi(2 x-3)+\frac{5}{16} \varphi(2 x-4)+\frac{1}{16} \varphi(2 x-5), \\
& \varphi_{5,-4}(x)=\left\{\begin{array}{cc}
\frac{(16 x+4)^{4}}{24}-\frac{5(16 x+4)^{3}}{6}+\frac{25(16 x+4)^{2}}{4}- & \\
\frac{125(16 x+4)}{6}+\frac{625}{24} & 0 \leq x<1 / 16, \\
0 & \text { else, }
\end{array}\right.
\end{aligned}
$$




$$
\begin{aligned}
& \varphi_{5,-3}(x)=\left\{\begin{array}{cc}
-\frac{(16 x+3)^{4}}{6}+\frac{5(16 x+3)^{3}}{2}-\frac{55(16 x+3)^{2}}{4}+ & 0 \leq x<1 / 16, \\
\frac{65(16 x+3)}{2}-\frac{655}{24} & 1 / 16 \leq x<1 / 8, \\
\frac{(16 x+3)^{4}}{24}-\frac{5(16 x+3)^{3}}{6}+\frac{25(16 x+3)^{2}}{4}- & \text { else, }
\end{array}\right. \\
& \varphi_{5,-2}(x)=\left\{\begin{array}{cc}
\frac{(16 x+2)^{4}}{4}-\frac{5(16 x+2)^{3}}{2}+\frac{35(16 x+2)^{2}}{4}- & \\
\frac{25(16 x+2)}{2}+\frac{155}{24} & 0 \leq x<1 / 16, \\
-\frac{(16 x+2)^{4}}{6}+\frac{5(16 x+2)^{3}}{2}-\frac{55(16 x+2)^{2}}{4}+ & 1 / 16 \leq x<1 / 8, \\
\frac{65(16 x+2)}{2}-\frac{655}{24} & 1 / 8 \leq x<3 / 16, \\
\frac{(16 x+2)^{4}}{24}-\frac{5(16 x+2)^{3}}{6}+\frac{25(16 x+2)^{2}}{4}- & \text { else, }
\end{array}\right. \\
& \varphi_{5,-1}(x)=\left\{\begin{array}{cc}
-\frac{(16 x+1)^{4}}{6}+\frac{5(16 x+1)^{3}}{6}-\frac{5(16 x+1)^{2}}{4}+ & 0 \leq x<1 / 16, \\
\frac{5(16 x+1)}{6}-\frac{5}{24} & 1 / 16 \leq x<1 / 8, \\
\frac{(16 x+1)^{4}}{4}-\frac{5(16 x+1)^{3}}{2}+\frac{35(16 x+1)^{2}}{4}- & 1 / 8 \leq x<3 / 16, \\
-\frac{25(16 x+1)}{2}+\frac{155}{24} & 3 / 16 \leq x<1 / 4, \\
\frac{(16 x+1)^{4}}{6}+\frac{5(16 x+1)^{3}}{2}-\frac{55(16 x+1)^{2}}{4}+ & \text { else, }
\end{array}\right. \\
& \varphi_{5,12}(x)=\left\{\begin{array}{cc}
-\frac{(16 x-12)^{4}}{6}+\frac{5(16 x-12)^{3}}{2}-\frac{55(16 x-12)^{2}}{4}+ & \\
\frac{65(16 x-12)}{2}-\frac{655}{24} & 15 / 16 \leq x<1, \\
\frac{(16 x-12)^{4}}{4}-\frac{5(16 x-12)^{3}}{2}+\frac{35(16 x-12)^{2}}{4}- & \\
\frac{25(16 x-12)}{2}+\frac{155}{24} & 7 / 8 \leq x<15 / 16, \\
-\frac{(16 x-12)^{4}}{6}+\frac{5(16 x-12)^{3}}{6}-\frac{5(16 x-12)^{2}}{4}+ & 13 / 16 \leq x<7 / 8, \\
\frac{5(16 x-12)}{6}-\frac{5}{24} & 12 / 16 \leq x<13 / 16, \\
\frac{(16 x-12)^{4}}{24} & \text { else, },
\end{array}\right.
\end{aligned}
$$$$
\varphi_{5,13}(x)=\left\{\begin{array}{cc}
\frac{(16 x-13)^{4}}{4}-\frac{5(16 x-13)^{3}}{2}+\frac{35(16 x-13)^{2}}{4}- & \\
\frac{25(16 x-13)}{2}+\frac{155}{24} & 15 / 16 \leq x<1, \\
-\frac{(16 x-13)^{4}}{6}+\frac{5(16 x-13)^{3}}{6}-\frac{5(16 x-13)^{2}}{4}+ & \\
\frac{5(16 x-13)}{6}-\frac{5}{24} & 7 / 8 \leq x<15 / 16, \\
\frac{(16 x-13)^{4}}{24} & 13 / 16 \leq x<7 / 8, \\
0 & \text { else, }
\end{array}\right.
$$$$
\varphi_{5,14}(x)=\left\{\begin{array}{cc}
-\frac{(16 x-14)^{4}}{6}+\frac{5(16 x-14)^{3}}{6}-\frac{5(16 x-14)^{2}}{4}+ & \\
\frac{5(16 x-14)}{6}-\frac{5}{24} & 15 / 16 \leq x<1, \\
\frac{(16 x-14)^{4}}{24} & 7 / 8 \leq x<15 / 16, \\
0 & \text { else },
\end{array}\right.
$$ 


$$
\varphi_{5,15}(x)=\left\{\begin{array}{cc}
\frac{(16 x-15)^{4}}{24} & 15 / 16 \leq x<1, \\
0 & \text { else } .
\end{array}\right.
$$

Further, of (2.6) and (2.7) and by compact support in the range [0,2m-1] we obtain:

$$
\psi_{5}(x)=\left\{\begin{array}{cc}
\frac{1}{8709120} x^{4} & 0 \leq x<1 / 2, \\
-\frac{73}{1244160} x^{4}+\frac{1}{8505} x^{3}-\frac{1}{11340} x^{2}+ & 1 / 2 \leq x<1, \\
\frac{1}{34020} x-\frac{1}{272160} & \vdots \\
\vdots & 8 \leq x<17 / 2, \\
\frac{503}{8709120} x^{4}-\frac{1069}{544320} x^{3}+\frac{72701}{2903040} x^{2}- & 17 / 2 \leq x<9, \\
\frac{1236079}{8709120} x+\frac{21016259}{69672960} & \text { else }, \\
\frac{1}{8709120} x^{4}-\frac{1}{241920} x^{3}+\frac{1}{17920} x^{2}- & \\
\frac{3}{8960} x+\frac{27}{35840} & \\
0 & \\
\psi(x)=\sum_{k=0}^{13} q_{k} \varphi(2 x-k) . &
\end{array}\right.
$$

\subsection{Function approximation using scaling function}

For any positive integer $M=j_{0}$, a function $f(x)$ defined over [0,1] may be represented by B-spline scaling functions as:

$$
f(x)=\sum_{k=-4}^{2^{M}-1} s_{k} \varphi_{M, k}=S^{T} \Phi_{M},
$$

where

$$
\begin{gathered}
S=\left[s_{-4}, s_{-3}, \ldots, s_{2^{M}-1}\right], \\
\Phi_{M}=\left[\Phi_{M,-4}, \Phi_{M,-3}, \ldots, \Phi_{M, 2^{M}-1}\right],
\end{gathered}
$$

with

$$
s_{k}=\int_{0}^{1} f(x) \tilde{\varphi}_{M, k}(x) d x, \quad k=-4,-3, \ldots, 2^{M}-1,
$$

where $\tilde{\varphi}_{M, k}(x)$ are dual functions of $\varphi_{M, k}(x)$,

$$
\widetilde{\Phi}=T_{\Phi} \Phi, \quad T_{\Phi}=\left(P_{M}\right)^{-1} .
$$

These can be obtained by linear combinations of $\varphi_{M, k}(x), k=-4,-3, \ldots, 2^{M}-1$, as follows. Let $\tilde{\Phi}_{M}$ be the dual functions of $\Phi_{M}$ given by:

$$
\tilde{\Phi}_{M}=\left[\tilde{\varphi}_{M,-4}, \tilde{\varphi}_{M,-3}, \ldots, \tilde{\varphi}_{M, 2^{M}-1}\right],
$$

using (2.8) we get:

$$
\int_{0}^{1} \tilde{\Phi}_{M} \Phi_{M}^{T} d x=I
$$

where $I$ is $\left(2^{M}+4\right) \times\left(2^{M}+4\right)$ identity matrix, let:

$$
P_{M}=\int_{0}^{1} \Phi_{M} \Phi_{M}^{T} d x
$$

the entries of the matrix $P_{M}$ is calculated from:

$$
\int_{0}^{1} \varphi_{M, i}(x) \varphi_{M, j}(x) d x
$$


from (2.8) and (2.9) we get:

$$
\tilde{\Phi}_{M}=\left(P_{M}\right)^{-1} \Phi_{M}
$$

Furthermore, a function $f(x)$ defined over $[0,1]$ may be represented by BSW as:

$$
f(x)=\sum_{k=-4}^{2^{j}-1} c_{m, k} \varphi_{5, k}(x)+\sum_{j=5}^{\infty} \sum_{k=-4}^{2^{j}-5} d_{j, k} \psi_{j, k}(x),
$$

if the infinite serie is truncated at $M$, then can be written as:

$$
f(x) \simeq \sum_{k=-4}^{15} c_{m, k} \varphi_{5, k}(x)+\sum_{j=5}^{M} \sum_{k=-4}^{2^{j}-5} d_{j, k} \psi_{j, k}(x)=C^{T} \Psi(x),
$$

where $\varphi_{5, k}$ and $\psi_{j, k}$ are scaling and wavelets functions, respectively and $C$ and $\Psi$ are $\left(2^{M}+4\right) \times 1$ vectors given by:

$$
\begin{gathered}
C=\left[c_{-4}, c_{-3}, \ldots, c_{15}, d_{5,-4}, d_{5,-3}, \ldots, d_{5,7}, \ldots, d_{M,-M+1}, \ldots, d_{2^{M},-5}\right], \\
\Psi=\left[\varphi_{5,-4}, \varphi_{5,-3}, \ldots, \varphi_{5,15}, \psi_{5,-4}, \psi_{5,-3}, \ldots, \psi_{5,7}, \ldots, \psi_{M,-M+1}, \ldots, \psi_{M, 2^{M}-5}\right],
\end{gathered}
$$

with

$$
\begin{gathered}
c_{k}=\int_{0}^{1} f(x) \tilde{\varphi}_{5, k}(x) d x, \quad k=-4,-3, \ldots 15, \\
d_{j, k}=\int_{0}^{1} f(x) \tilde{\psi}_{j, k}(x) d x, \quad j=5,4, \ldots M, \quad k=-4,-3, \ldots, 2^{j}-5 .
\end{gathered}
$$

These can be obtained by linear combinations as follows:

$$
\begin{gathered}
\Phi=\left[\varphi_{5,-4}(x), \varphi_{5,-3}(x), \ldots, \varphi_{5,15}(x)\right]^{T}, \\
\Psi=\left[\psi_{5,-4}(x), \psi_{5,-3}(x), \ldots, \psi_{M, 2^{M}-5}(x)\right]^{T} .
\end{gathered}
$$

For numerical solving of (1.1) we should choose a finite dimensional family of functions so that the exact solution may be estimated by them. Methods that use this strategic technique are called projection methods, because the exact solution of equation is projected onto the space with finite dimensions. One of the most famous for these methods is collocation method. We choose a sequence of finite dimensional subspaces $X_{n} \subset L^{2}(\mathbb{R})$ for $n \geq 1$, with $X_{n}$ having dimension $d_{n}$. Assume that $X_{n}$ has a basis of the form $\varphi_{1}, \varphi_{2}, \ldots, \varphi_{d}$ with $d \equiv d_{n}$ for notational simplicity and $\varphi_{n}$ is a function which belongs to $X_{n}$, so that we can write it as $\phi(s) \approx \phi_{n}(s)=\sum_{i=1}^{d} c_{i} \varphi_{i}(s)$. By substitution into (1.1) we have:

$$
r_{n}(x)=\int_{a}^{b} k(x, s) \phi_{n}(s) d s-f(x)=\int_{a}^{b} k(x, s) \sum_{i=1}^{d} c_{i} \varphi_{i}(s) d s-f(x),
$$

where $r_{n}$ is called the residual in the approximation of the equation when using $\phi \approx \phi_{n}$. In the operator form we have $r_{n}=k \phi_{n}-f$. Now to determine the unknown coefficients $\left\{c_{i}\right\}_{i=1}^{d}$ we impose the following requirements:

$$
r_{n}\left(x_{i}\right)=0, \quad i=1,2, \ldots, d,
$$

where $x_{i}$ are the collocation node points. These coefficients are determined uniquely if and only if $\varphi_{i}(x)$ are being independent. In this paper, we use QBSW family which are SO wavelet basis, so that $\phi_{n}(s)=\sum_{i=1}^{d} c_{i} \varphi_{i}(s)$ is uniquely determined. 


\section{The method of regularization}

Assuming that a solution exists to the linear ill-posed problem (1.1) which can always be written in the generic form $K \phi=f$. With modify (1.1) we can write:

$$
\int_{a}^{b} k(x, s) \phi(s) d s+\gamma \phi(x)=f(x),
$$

where $\gamma$ is known as the regularization parameter and (3.1) is an FK2 whose solution, denoted by $\phi_{\gamma}(x)$, can be found in [13]. These equations may be written as

$$
\phi(K+\gamma I)=f .
$$

Substituting $\phi_{\gamma}(x)$ for $\phi(x)$ in (1.1), we get:

$$
\int_{a}^{b} k(x, s) \phi_{\gamma}(s) d s=f_{\gamma}(x) .
$$

If $\left\|f(x)-f_{\gamma}(x)\right\| \leq \delta$ where $\delta$ is a preassigned quantity representing the tolerance of error, then the function $\phi_{\gamma}(x)$ is considered an acceptable approximate solution to (1.1) that we proof this task in error consideration section. Thus, if the kernel $k(x, s)$ is discontinuous along a curve $s=g(x)$ and the discontinuity is finite, then an FK1 can be changed to an FK2, as follows:

$$
f(x)=\int_{a}^{b} k(x, s) \phi(s) d s=\int_{a}^{g(x)} k(x, s) \phi(s) d s+\int_{g(x)}^{b} k(x, s) \phi(s) d s,
$$

and differentiating both sides with respect to $x$, we get:

$$
\begin{gathered}
f^{\prime}(x)=\int_{a}^{b} \frac{\partial k(x, s)}{\partial x} \\
=\int_{a}^{b} \frac{\partial k(x, s)}{\partial x} \phi(s) d s+S(x) \phi(g(x)) g^{\prime}(x),
\end{gathered}
$$

where $k\left(x, g(x)_{-}\right)$and $k\left(x, g(x)_{+}\right)$are defined everywhere in $(a, b)$, and the difference $S(x)=k\left(x, g(x)_{-}\right)-k\left(x, g(x)_{+}\right)$. On dividing the above equation by $S(x) g^{\prime}(x)$ and replacing $x$ by $x=g^{-1}(y)$, we obtain an FK2. Now, the following theorem can be stated.

Theorem 3.1. If $k(x, s)$ is bounded in the domain $\Omega=[a, b] \times[a, b]$ and continuous except on the curve $s=g(x)$, where $g(x)$ has a nonzero continuous derivative in $[a, b]$, with $g(a)=a$, and $g(b)=b$ and

(i) $S(x) \in C[a, b]$,

(ii) $k_{x}(x, s)$ is real and exists in $\Omega$,

(iii) $f(x)$ and $f^{\prime}(x)$ are continuous in $[a, b]$ and

the quantity

$$
\left|\frac{k_{x}(x, s)}{S(x) g^{\prime}(x)}\right|_{x=g^{-1}(y)},
$$

does not vanish in $\Omega$, then an FK1 can be changed into the following FK2 as:

$$
\int_{a}^{b}\left|\frac{k_{x}(x, s)}{S(x) g^{\prime}(x)}\right|_{x=g^{-1}(y)} \phi(s) d s+\phi(y)=\left|\frac{f^{\prime}(x)}{S(x) g^{\prime}(x)}\right|_{x=g^{-1}(y)} .
$$

Proof. See [4].

If $k(x, s)$ is continuous in $\Omega$, but if $\frac{\partial^{n} k(x, s)}{\partial x^{n}}$ for some $n$ has a finite discontinnuity at $s=g(x)$, then the theorem can be generalized. In order to solve (3.1) we consider:

$$
w(x)=\int_{a}^{b} k(x, s) \phi(s) d s,
$$


then, we first approximate $\phi(s)$ and $w(s)$ as:

$$
\begin{gathered}
\phi(s)=C^{T} \Psi(s), \\
w(x)=\int_{a}^{b} k(x, s) C^{T} \Psi(s) d s,
\end{gathered}
$$

where $C^{T} \Psi(s)$ is defined in (2.10) and $C$ is $\left(2^{M}+4\right) \times 1$ unknown vector. We can approximate (3.3) using quadrature Newton-Cotes (NC) integration techniques as:

$$
w(x)=\int_{a}^{b} k(x, s) C^{T} \Psi(s) d s=\sum_{i=1}^{n} \omega_{i} k\left(x, s_{i}\right) C^{T} \Psi\left(s_{i}\right),
$$

where $\omega_{i}$ and $s_{i}$ are weight and nodes of NC method. From (3.1) and (3.2), we get:

$$
w(x)+\gamma \phi(x)=f(x),
$$

to find the solution $\phi(x)$ we first collocate in $x_{i}=(2 i-1) /\left(2^{M+2}-2\right), i=1, \ldots, 2^{M+1}-1$, the resulting equation generates $2^{M+1}-1$ algebraic equations and the total unknowns for vector $C$ in (3.4) is $2^{M+1}+1$.

\section{Error and convergence consideration}

Theorem 4.1. If $\gamma$ regularization parametr and $\phi_{\gamma}(x)$ is approximate solution in

$$
\int_{a}^{b} k(x, s) \phi(s) d s+\gamma \phi(x)=f(x),
$$

and

then

$$
\int_{a}^{b} k(x, s) \phi_{\gamma}(s) d s=f_{\gamma}(x)
$$

$$
\left\|f(x)-f_{\gamma}(x)\right\| \leq \delta
$$

where $\delta$ is a known bound on the measurement error.

Proof. We can write FK2 equation in the operator form as:

$$
(k+\gamma I) \phi_{\gamma}=f,
$$

since, the operator $(K+\gamma I)$ has a bounded inverse then, the problem of solving the equation is well-posed. This FK2 equation have unique solution

$$
\phi_{\gamma}=(k+\gamma I)^{-1} f,
$$

from this we see that $\gamma \phi_{\gamma}=f-k \phi_{\gamma}$ and we may in passing to the limit as:

$$
\lim _{\gamma \rightarrow 0}\left\|\phi_{\gamma}-k^{\Lambda} f\right\|^{2}=0,
$$

that $k^{\Lambda}=(k+\gamma I)^{-1}$ and the vectors $\left\{\phi_{\gamma}\right\}$ are therefore genuine approximations to $k^{\Lambda}$ in the sense that $\phi_{\gamma} \rightarrow k^{\Lambda} f$ as $\gamma \longrightarrow 0$ and insomuch for each $\gamma>0$ the operator $k^{\Lambda}$ is bounded then, the approximation $\phi_{\gamma}$ depends continuously on $f$.

According to the regularization parameter $\gamma$ and approximation $\phi_{\gamma}(x)$ we can consider estimate $f^{\delta}$ of $f$ where $\delta$ is a known bound on the measurement error and with putting $f^{\delta}$ instead true function, we obtain the approximation form:

$$
\phi_{\gamma}^{\delta}=(k+\gamma I)^{-1} f^{\delta},
$$

with (4.1) and (4.2) we will have:

$$
\begin{aligned}
& \phi_{\gamma}^{\delta}-\phi_{\gamma}=(k+\gamma I)^{-1}\left(f^{\delta}-f\right), \\
& \left\|\phi_{\gamma}^{\delta}-\phi_{\gamma}\right\|^{2}=\left\langle(k+\gamma I)^{-1}\left(f^{\delta}-f\right),(k+\gamma I)^{-1}\left(f^{\delta}-f\right)\right\rangle,
\end{aligned}
$$


and

$$
\left\|(k+\gamma I)^{-1}\right\| \leq 1 / \gamma
$$

then

$$
\left\|\phi_{\gamma}^{\delta}-\phi_{\gamma}\right\| \leq \delta / \sqrt{\gamma}
$$

Then with select a suitable regularization parameter $\gamma=\gamma(\delta)$, we will have:

$$
\left\|\phi_{\gamma(\delta)}^{\delta}-k^{\Lambda} f\right\| \leq\left\|\phi_{\gamma(\delta)}^{\delta}-\phi_{\gamma(\delta)}\right\|+\left\|\phi_{\gamma(\delta)}-k^{\Lambda} f\right\| \leq \delta / \sqrt{\gamma(\delta)}+\left\|\phi_{\gamma(\delta)}-k^{\Lambda} f\right\|,
$$

if $\delta$ colse to zero then $\delta / \sqrt{\gamma(\delta)}$ close to zero and $\phi_{\gamma(\delta)} \rightarrow k^{\Lambda} f$.

With minimize an augmented Least Squares (LS) function $t(x)=\sum_{j=0}^{m} a_{j} t_{j}(x)$ that $t_{j}(x)$ known functions and $a_{j}$ are unknown coefficients, we will have:

$$
\begin{array}{r}
\mathbf{L S}_{\gamma}\left(a_{0}, a_{1}, \ldots, a_{m}\right)=\sum_{i=0}^{n}\left(\left(\sum_{j=0}^{m} a_{j} t_{j}\left(x_{i}\right)\right)-\varphi_{i}\right)^{2} \\
=\left\|k t-f^{\delta}\right\|^{2}+\gamma\|t\|^{2}
\end{array}
$$

If we consider linear function $t(x)=a_{0}+a_{1} x$ any minimizer of (4.3) must satisfy

$$
\left.\frac{d}{d x}\left\{\left\|k\left(a_{0}+a_{1} x\right)-f^{\delta}\right\|^{2}+\gamma\left\|a_{0}+a_{1} x\right\|^{2}\right\}\right|_{x=0}=0
$$

expressing the squared norms in terms of the inner product and expanding the quadratic forms this is equivalent to

$$
\left\langle k a_{0}-f^{\delta}, k a_{1}\right\rangle+\gamma\left\langle a_{0}, a_{1}\right\rangle=0,
$$

or

$$
\begin{gathered}
\left\langle(k+\gamma I) a_{0}-f^{\delta}, a_{1}\right\rangle=0, \\
(k+\gamma I) a_{0}=f^{\delta},
\end{gathered}
$$

substituting (4.4) in (4.2), we will get:

$$
\phi_{\gamma}^{\delta}=(k+\gamma I)^{-1} f^{\delta}=(k+\gamma I)^{-1}(k+\gamma I) a_{0},
$$

for the solution of $k \phi=f$ choose the regularization parameter so that the size of the residual $r(\gamma)=\left\|k \phi_{\gamma}^{\delta}-f^{\delta}\right\|$ is the same as the error level in the data and the vector $\phi$ of minimum norm satisfying the requirement

$$
\left\|k \phi-f^{\delta}\right\| \leq \delta
$$

Now if $P$ is the orthogonal projector of Hilbert space, we can write:

$$
\begin{aligned}
& P(k+\gamma I) \phi_{\gamma}^{\delta}=P f^{\delta}, \\
& P\left(\gamma \phi_{\gamma}^{\delta}\right)+P\left(k \phi_{\gamma}^{\delta}\right)=P f^{\delta}, \\
& k \phi_{\gamma}^{\delta}=f^{\delta}-\gamma \phi_{\gamma}^{\delta}, \\
& \left\|k \phi_{\gamma}^{\delta}-f^{\delta}\right\|=\left\|\gamma \phi_{\gamma}^{\delta}\right\|=\left\|P f^{\delta}-P k \phi_{\gamma}^{\delta}\right\|,
\end{aligned}
$$

if $\gamma \longrightarrow 0$ given:

$$
\lim _{\gamma \rightarrow 0^{+}} r(\gamma)=\left\|P f^{\delta}-P k \phi_{\gamma}^{\delta}\right\|=\left\|P f^{\delta}-P f\right\| \leq\left\|f^{\delta}-f\right\| \leq \delta,
$$

and

$$
\lim _{\gamma \rightarrow \infty} r(\gamma)=\left\|f^{\delta}\right\|>\delta,
$$

the choice $\gamma(\delta)$ as given leads to a regular scheme for approximating $k^{\Lambda} f$, that is

$$
f_{\gamma(\delta)}^{\delta} \rightarrow k^{\Lambda} f \quad \text { as } \quad \delta \rightarrow 0
$$


Theorem 4.2. Assume that $f \in C^{5}[0,1]$ is represented by quartic B-spline wavelets as :

$$
d_{j, k}=\int_{0}^{1} f(x) \tilde{\psi}_{j, k}(x) d x,
$$

where $\psi$ has 5 vanishing moments, then

$$
\left|d_{j, k}\right| \leq \mu \varepsilon \frac{2^{-6 j}}{5 !}
$$

where $\mu=\max \left|f^{(5)}(t)\right|_{[0,1]}$ and $\varepsilon=\int_{0}^{1}\left|x^{5} \tilde{\psi}_{j}(x)\right| d x, j=5, \ldots, M, k=-4, \ldots, 2^{j}-5$.

Proof. We approximate the function $f \in C^{5}[0,1]$ at arbitrary point $x_{0} \in[0,1]$ as:

$$
f(x) \simeq \sum_{p=0}^{4} \frac{\left(x-x_{0}\right)^{p}}{p !} f^{(p)}\left(x_{0}\right)+\frac{\left(x-x_{0}\right)^{5}}{5 !} f^{(5)}(\xi) \quad, \quad \xi \in D_{f},
$$

$f(x)$ may be represented by quartic B-spline wavelets and with substituting (4.5) we will get:

$$
d_{j, k} \leq \int_{0}^{1} \tilde{\psi}_{j, k}(x)\left(\sum_{p=0}^{4} \frac{\left(x-x_{0}\right)^{p}}{p !} f^{(p)}\left(x_{0}\right)+\frac{\left(x-x_{0}\right)^{5}}{5 !} f^{(5)}(\xi)\right) d x,
$$

the actual coordinate position $x$ is related to $x_{j}$ according to $x_{j}=2^{j} x_{0}$ and have $t=$ $2^{j} x-x_{j}$ then

$$
d_{j, k} \leq \sum_{p=0}^{5} \frac{f^{(p)}\left(x_{j} / 2^{j}\right)}{2^{j(p+1)} p !} \int_{0}^{1} t^{p} \tilde{\psi}(t) d t+\int_{0}^{1} \frac{\left(x-x_{0}\right)^{5}}{5 !} f^{(5)}(\xi) \tilde{\psi}_{j, k}(x) d x,
$$

because $\psi$ has 5 vanishing moments then:

$$
\sum_{p=0}^{5} \frac{f^{(p)}\left(x_{j} / 2^{j}\right)}{2^{j(p+1)} p !} \int_{0}^{1} t^{p} \tilde{\psi}(t) d t=0
$$

thus we will get

$$
d_{j, k} \leq \frac{f^{(5)}(\xi)}{5 !} 2^{-6 j} \int_{0}^{1} x^{5} \tilde{\psi}_{j, k}(x) d x=\mu \frac{2^{-6 j}}{5 !} \varepsilon
$$

Theorem 4.3. Suppose $f$ is represented by quartic B-spline scaling function $\varphi$ as:

$$
c_{k}=\int_{0}^{1} f(x) \tilde{\varphi}_{5, k}(x) d x, \quad k=-4,-3, \ldots 15,
$$

and the biorthogonal projection $P_{j}: L_{2}(\mathbb{R}) \longrightarrow V_{j}$ of function $f \in L_{2}(\mathbb{R})$ on to the space $V_{j}$ then $P_{j} f$ converges to $f$.

Proof. Because dual scaling function $\tilde{\varphi}$ such that

$$
\langle\varphi(x), \tilde{\varphi}(x-k)\rangle=\delta_{k} \quad, \quad k \in \mathbb{Z},
$$

given scaling function $\varphi$, the biorthogonal projection $P_{j}$ of function $f$ is given by

$$
P_{j} f=\sum_{k \in \mathbb{Z}}\left\langle f, \tilde{\varphi}_{j, k}\right\rangle \varphi_{j, k}
$$

if $\Phi_{j}$ represents polynomials in $p_{d-1}$, the following error estimate is well-known

$$
\left\|f-P_{j} f\right\| \leq 2^{-j s}\|f\| \quad, \quad f \in H^{s}(\Omega), \quad 0 \leq s \leq d,
$$


Table 1. Absolute Error for Example 1

\begin{tabular}{cccccc}
\hline \hline & \multicolumn{2}{c}{$(\mathbf{M}=\mathbf{5})$} & \multicolumn{2}{c}{$(\mathbf{M}=\mathbf{6})$} & \multirow{2}{*}{ Method of $[9]$} \\
\cline { 2 - 5 }$s_{i}$ & $\gamma=0.01$ & $\gamma=0.001$ & $\gamma=0.01$ & $\gamma=0.001$ & \\
\hline 0.1 & $3.3(-4)$ & $2.8(-4)$ & $3.5(-5)$ & $1.9(-6)$ & $5.0(-4)$ \\
0.2 & $4.6(-4)$ & $4.6(-5)$ & $4.9(-5)$ & $6.0(-6)$ & $3.3(-4)$ \\
0.3 & $6.4(-5)$ & $7.1(-5)$ & $5.3(-6)$ & $7.5(-6)$ & $4.6(-4)$ \\
0.4 & $2.8(-5)$ & $8.6(-5)$ & $3.2(-6)$ & $3.2(-7)$ & $5.9(-4)$ \\
0.5 & $3.5(-5)$ & $6.5(-6)$ & $5.5(-6)$ & $6.6(-7)$ & $2.2(-3)$ \\
0.6 & $6.3(-5)$ & $4.0(-6)$ & $2.2(-7)$ & $5.7(-7)$ & $8.3(-4)$ \\
0.7 & $4.5(-5)$ & $7.1(-5)$ & $6.0(-7)$ & $9.0(-6)$ & $7.8(-4)$ \\
0.8 & $1.3(-6)$ & $3.3(-6)$ & $4.7(-7)$ & $5.5(-7)$ & $4.8(-4)$ \\
0.9 & $2.7(-6)$ & $6.8(-6)$ & $8.2(-7)$ & $4.3(-7)$ & $8.5(-4)$ \\
\hline
\end{tabular}

moreover, $H^{s}(\Omega)$ denotes the standard Sobolev norm of weakly differential of order up to $s \in N$ normed by

if $\mu=\sup \|f\|_{s}$ then

$$
\|f\|_{s}^{2}=\sum_{m=0}^{s}\left\|\partial^{m} f\right\|^{2}
$$

$$
\left\|f-P_{j} f\right\| \leq 2^{-j s} \mu,
$$

if the number $j$ is large and or the bound $\mu$ small, we will have:

$$
\left\|f-P_{j} f\right\| \rightarrow 0 \quad \text { or } \quad P_{j} f \rightarrow f
$$

consider that $e_{j}(x)$ be error of approximation in space $V_{j}$, then:

$$
\left|e_{j}(x)\right|=O\left(2^{-5 j}\right) .
$$

Thus, order of error depend on the level $j$. Obviously, for larger level of $j$, the error of approximation will be smaller.

\section{Illustrative examples}

The numerical experiments are implemented in Maple 15 software. The programs are executed on a PC with $2.00 \mathrm{GHz}$ Intel Core 2 dual processor with 2 GB RAM. In illustrative examples, to show the accuracy and efficiency of the described method, we presented numerical examples, then we compared the results of our methods with the results of some other methods. The matrix sizes for the QBSW in $M=j_{0}=4$ are of order $\left(2^{j_{0}}+1\right)$ and $\left(2^{j_{0}+1}+1\right)$ were $65 \times 65$ and $129 \times 129$, respectively, for $M=5$ and $M=6$.

Example 5.1. Consider the FK1 integral equation

$$
\int_{0}^{1} \mathrm{e}^{x s} \phi(s) d s=\frac{\mathrm{e}^{x+1}-1}{x+1}
$$

with exact solution $\phi(x)=\exp (x)$. The absolute error at the particular grid points is tabulated in Table 1, that shows a comparison between our method together with method in [9].

Example 5.2. Consider the Fk1 integral equation

$$
\int_{0}^{1} \sqrt{x^{2}+s^{2}} \phi(s) d s=1 / 3\left(1+x^{2}\right)^{3 / 2}-1 / 3 x^{3},
$$

with exact solution $\phi(x)=x$, the absolute error is tabulated in Table 2 and comparison with method in [10]. 
Table 2. Absolute Error for Example 2

\begin{tabular}{cccccc}
\hline \hline & \multicolumn{2}{c}{$(\mathbf{M}=\mathbf{5})$} & \multicolumn{2}{c}{$(\mathbf{M = 6 )}$} & \multirow{2}{*}{ Method of $[\mathbf{1 0 ]}$} \\
\cline { 2 - 5 }$s_{i}$ & $\gamma=0.01$ & $\gamma=0.001$ & $\gamma=0.01$ & $\gamma=0.001$ & \\
\hline 0.1 & $4.6(-5)$ & $4.6(-6)$ & $6.2(-6)$ & $2.7(-7)$ & $1.5(-1)$ \\
0.2 & $5.5(-5)$ & $7.0(-6)$ & $7.7(-6)$ & $5.6(-7)$ & $4.2(-1)$ \\
0.3 & $4.9(-5)$ & $1.8(-6)$ & $8.3(-6)$ & $3.9(-7)$ & $1.1(-1)$ \\
0.4 & $6.7(-5)$ & $5.5(-6)$ & $5.2(-7)$ & $7.2(-7)$ & $6.2(-2)$ \\
0.5 & $8.1(-5)$ & $4.6(-6)$ & $2.9(-7)$ & $4.8(-7)$ & $3.3(-2)$ \\
0.6 & $2.2(-6)$ & $2.8(-6)$ & $4.4(-6)$ & $2.2(-7)$ & $2.3(-2)$ \\
0.7 & $3.9(-6)$ & $1.2(-7)$ & $3.0(-7)$ & $8.1(-6)$ & $2.8(-2)$ \\
0.8 & $7.6(-6)$ & $4.6(-6)$ & $2.7(-7)$ & $5.4(-7)$ & $8.9(-3)$ \\
0.9 & $2.5(-5)$ & $2.6(-7)$ & $4.1(-7)$ & $6.0(-7)$ & $1.5(-2)$ \\
\hline
\end{tabular}

Table 3. Absolute Error for Example 3

\begin{tabular}{cccccc}
\hline \hline & \multicolumn{2}{c}{$(\mathbf{M}=\mathbf{5})$} & \multicolumn{2}{c}{$(\mathbf{M}=\mathbf{6})$} & \multirow{2}{*}{ Method of $[\mathbf{1}]$} \\
\cline { 2 - 5 }$s_{i}$ & $\gamma=0.01$ & $\gamma=0.001$ & $\gamma=0.01$ & $\gamma=0.001$ & \\
\hline 0.1 & $6.6(-5)$ & $2.6(-6)$ & $3.2(-7)$ & $5.6(-7)$ & $5.3(-5)$ \\
0.2 & $3.9(-6)$ & $7.1(-6)$ & $2.2(-7)$ & $5.2(-7)$ & $1.3(-4)$ \\
0.3 & $7.1(-5)$ & $4.7(-6)$ & $7.1(-7)$ & $8.1(-7)$ & $2.2(-4)$ \\
0.4 & $8.2(-5)$ & $7.2(-6)$ & $8.3(-6)$ & $3.1(-7)$ & $3.0(-4)$ \\
0.5 & $2.2(-5)$ & $7.0(-6)$ & $6.5(-7)$ & $6.9(-7)$ & $5.9(-4)$ \\
0.6 & $4.5(-5)$ & $6.5(-6)$ & $8.7(-6)$ & $4.4(-7)$ & $3.8(-4)$ \\
0.7 & $4.0(-5)$ & $3.1(-6)$ & $3.9(-6)$ & $5.8(-6)$ & $5.5(-4)$ \\
0.8 & $9.4(-5)$ & $1.1(-6)$ & $6.1(-6)$ & $2.0(-7)$ & $7.2(-4)$ \\
0.9 & $3.7(-5)$ & $5.4(-6)$ & $5.7(-6)$ & $5.7(-7)$ & $8.9(-4)$ \\
\hline
\end{tabular}

Example 5.3. Consider the FK1 integral equation with exact solution $\phi(x)=x$,

$$
\int_{0}^{1} \sin (x s) \phi(s) d s=\frac{\sin x-x \cos x}{x^{2}},
$$

Table 3 shows comparison between the absolute error of our method with method in [1].

\section{Conclusion}

According to the cases mentioned in section error and convergence analysis, the method showed that the approximation method using B-spline wavelet method needs to be derivative of the fifth. According to the examples which solved the absolute error, the results obtained from the B-spline method are less. Although the Fredholm integral equation of the first kind is malignant but there was a systematic method of expression which turns it into an integral equation of the second kind ; in the mean while the use of quasi-orthogonal wavelets in the solution was very helpful. Moreover, because of semi-orthoganality and having been vanishing moments of B-spline wavelets, matrices in our method are sparse, thus we do not need large memory requirement and a high computational time.

\section{References}

[1] H. Adibi and P. Assari, Chebyshev wavelet method for numerical solution of Fredholm integral equation of the first kind, Math. Prob. Eng, 2010, Article ID 138408, 17 pp., 2010 . 
[2] K. Atkinson, The Numerical Solution of Integral Equations of the Second Kind, Cambridge University Press 2, 1997.

[3] C.K. Chui, L. Montefusco and L. Puccio, Wavelets, Theory algorithm and applications, Academic press, 1994.

[4] A. Cohen, Numerical Analysis of Wavelet Methods, New York, Academic Press, 2003.

[5] P.K. Kythe and P. Puri, Computational Methods for Linear Integral Equations, Springer Science, 2002.

[6] K. Maleknejad, T. Lotfi and K. Mahdiani, Numerical solution of first kind Fredholm integral equation with wavelets-Galerkin method (WGM) and wavelets precondition, Appl. Math. Comput. 186, 794-800, 2007.

[7] K. Maleknejad, T. Lotfi and Y. Rostami, Numerical Computational Method in Solving Fredholm Integral Equations of the Second Kind by Using Coifman Wavelet, Appl. Math. Comput. 186, 212-218, 2007.

[8] K. Maleknejad, H. Mesgarani and T. Nikazad, Wavelet-Galerkin Solutions For Fredholm Integral Equations of The Second Kind, Internat. J. Engng. Sci. 13, 75-80, 2002.

[9] K. Maleknejad and S. Sohrabi,Numerical solution of Fredholm integral equation of the first kind by using Legendre wavelets, Appl. Math. Comput. 186, 836-843, 2007.

[10] M.T. Rashed, Numerical solutions of the integral equations of the first kind, Appl. Math. Comput. 2-3 , 413-420, 2003.

[11] P.K. Sahu and S. Saha Ray, Numerical solutions for the system of Fredholm integral equations of second kind by a new approach involving semiorthogonal B-spline wavelet collocation method. Appl. Math. Comput. 234, 368-379, 2014.

[12] M. Shamsi and M. Razzaghi, Solution of Hallens integral equation using multiwavelets, Comput. Phys. Comm. C 168, 187-197, 2015.

[13] A.M. Wazwaz, The regularization method for Fredholm integral equations of the first kind, Comput. Math. Appl. 61 (10), 2981-2986, 2011.

[14] G.A. Zakeri and M. Navab, Sinc collocation approximation of non-smooth solution of a nonlinear weakly singular Volterra integral equation, J. Comput. Phys. 229, 6548-6557, 2010. 